\title{
«Modus et ratio visendi aegros» des Solothurner Arztes Johann Jakob Scharandaeus (1630-1682)
}

Von Joseph Schürmann-Roth

1550 bürgerte sich in Solothurn der Krämer Claudius Schirandin aus dem Aostatal ein. Sein Sohn Ludwig (*1591) war der erste Bürgerssohn, der Stadtarzt wurde. Als er 1636 an der Pest starb, hinterließ er einen Sohn Christoph (1611-1691), der ebenfalls Arzt war und von 1642 bis 1670 in politischen Ämtern nachgewiesen ist, und einen erst 6jährigen Knaben Johann Jakob (1630-1682). Dieser wurde vom Rat 1650 aus Amsterdam heimgerufen, um nach Christoph zurMatten (1606-1650) Stadtarzt zu werden ${ }^{1}$. Er blieb es bis zu seinem Tode am 6. Oktober 1682. Die Familie, in Solothurn Tscharandi genannt, hat der Stadt noch einige bedeutende Leute gestellt; der letzte Namensträger, ein Franziskaner, starb $1799^{2,3,4,5}$. Das Andenken an die Familie - wie auch das an die ebenfalls ausgestorbenen zurMatten - blieb erhalten durch die Benennung von Straßen nach ihren Namen, und in der Fastnachtszeit wird heute noch in Solothurn ein Tscharandi-Ball abgehalten.

Johann Jakob hat vielleicht zuerst in Montpellier Medizin studiert. Er schildert nämlich im IV. Kapitel seines Büchleins, mit dem wir uns im folgenden beschäftigen werden, eine Schiffsreise durch die «Säulen des Herkules» bis nach Texel in Holland. In Amsterdam ist 1649 seine erste Arbeit - wohl die Dissertation erschienen: «Ratio conservandae sanitatis ». ${ }^{6}$ Der Titel klingt auffallend ähnlich dem eines Buches, verfaßt von seinem Vorgänger Christoph zurMatten: «Tractatus de tuenda valetudine», das 1636 in Paris herausgekommen war. Ob J.J. Scharandaeus - wie die latinisierte Form des Familiennamens lautet - wirklich doktoriert hat, bleibt fraglich, denn 1670 bezeichnet er sich auf dem Titelblatt von «Modus et ratio visendi aegros» bloß als «Philosophus et Medicus».

Nach zwanzig Jahren Erfahrung in der Praxis nämlich setzte sich Johann Jakob Scharandaeus nochmals an den Schreibtisch und verfaßte dieses Büchlein von 280 Oktavseiten, gewidmet dem Abt von Bellelay, Johann Georg Schwaller (Abt 1666-1691), der vielleicht des Verfassers Altersgenosse, jedenfalls auch Solothurner Patrizierssohn war ${ }^{7}$. Nach Haller ${ }^{8}$ wurde es, von einem nicht genannten Bearbeiter mit Beispielen «ex Hofmanno et Roderico a Castro» erweitert, 1749 in Erfurt nochmals aufgelegt. Weder als Stadtarzt noch als Schriftsteller ist J. J.Scharandaeus so berühmt geworden, daß ihm die Ehre widerfahren wäre, von Leu im Allgemeinen Helvetischen Lexikon erwähnt zu werden wie 
Großvater, Vater, Brüder und Neffen. Wahrscheinlich hat Leus Solothurner Korrespondent andere Namensträger vorgezogen.

Mit seinem Buch über «Modus et ratio visendi aegros» wollte Scharandaeus jungen Kollegen Kenntnisse vermitteln, die ihnen an hohen Schulen nicht beigebracht wurden. Den Titel möchte man auf den ersten Blick mit «Untersuchungslehre» übersetzen. Hat man sich aber mit dem Inhalt etwas vertraut gemacht, so ist selbst «Über den Umgang mit Kranken» noch zu eng gefaßt. Scharandaeus wollte Erfahrungen allgemeiner Natur niederlegen, die er in zwanzig Jahren Tätigkeit gesammelt hatte, Erfahrungen weniger am Krankenbett - sonst hätte er ja «Observationes» als Titel wählen müssen - als vielmehr solche aus der Berufsausübung, und an einer Stelle läßt er sogar etwas von Reue und Bedauern darüber durchblicken, daß er sein Leben der Heilkunst geweiht habe. - Wie schon der Titel nicht ganz dem Inhalt entspricht, besteht auch in den Kapiteln nicht immer Übereinstimmung zwischen der Überschrift und dem, was gesagt wird. Polemik gegen die Alchimisten ist in zwei weit auseinanderliegenden Kapiteln geradezu versteckt; im Titel erscheint jeweilen keine Andeutung davon.

Des Scharandaeus Quellen waren mannigfacher Art. Als «Philosophus» mußte er die lateinischen Klassiker beherrschen. Viele entlegene Autorennamen, die er zitiert, lassen sich auf die verschiedenen Werke von Cicero und Seneca und auf die «Historia naturalis» des Plinius zurückverfolgen; Plutarch wird vielfach herangezogen; Plato und Xenophon spielen eine gewisse Rolle; Ovid und Horaz, Catull und Tibull, Plautus und Terenz müssen dem Verfasser geläufig gewesen sein. Zahlreich sind Zitate, zumal gereimte, bei denen der Dichter nicht angegeben ist, weil Scharandaeus wohl voraussetzte, ein gebildeter Leser werde die Herkunft kennen. Hippokrates und Galen waren ihm sehr gut bekannt, vermutlich aus Kommentaren wie dem von Hollerius ${ }^{9}$ und Duretus ${ }^{10}$. Auf des Johannes Langius «Epistolae medicinales» ${ }^{11}$ geht wohl die kritische Einstellung des Verfassers zurück. Die Portugiesen Valescus Tarentinus ${ }^{12}$ und Rodericus a Castro ${ }^{13}$ - in Montpellier hoch geschätzt - galten ihm viel. - Es erübrigt sich, alle Quellen aufzuzählen; aber aufschlußreich mag doch der Hinweis darauf sein, was von Scharandaeus gerade nicht zitiert wird, obschon es ihm der Zeit nach hätte bekannt sein sollen: vorab Felix Platters «Praxis» ${ }^{14}$, von der auf der Höhe des Wirkens von Scharandaeus noch zwei Auflagen erschienen, Johann Jakob Wepfers «Apoplexia » ${ }^{15}$ von 1658 oder das «Pantheon hygiasticum hippocraticohermeticum» (Pruntrut 1628) des fürstbischöflich-baslerischen Leibarztes Claudius Deodatus, der - wie andere zu seiner Zeit ebenfalls - aufgrund von Hippokrates und Paracelsus ein Leben von 120 Jahren in Aussicht stellen wollte. Man darf sich fragen, ob Scharandaeus nach seiner Heimkehr in die Vaterstadt noch 
weitere Literatur angeschafft habe. Als überzeugter Galeniker und Hippokratiker könnte er Bedenken gegen die neue Auffassung über den Blutkreislauf noch von Montpellier mitgebracht haben.

Zunächst untersucht Scharandaeus sehr wortreich, ob Ärzte überhaupt nötig seien. Weil sie von Gesundheit etwas verstehen und imstande sind, dieses höchste irdische Gut zu überwachen, wird die Frage bejaht. Die Eigenschaften des wahren Arztes werden denen der Apotheker, Chirurgen, Bader und Hebammen gegenübergestellt. Auch anderswo im Buche werden die Apotheker nicht gut wegkommen.

Wie soll ein so beschaffener Arzt ausgebildet werden? Scharandaeus skizziert den Werdegang, wie er ihn offensichtlich selber erlebt hatte: Philosophie vor allem andern, dann Anatomie und Materia medica, und darüber hinaus noch Kenntnisse auf irgendeinem andern Gebiet, damit sich der Arzt in einem Kreise gebildeter Leute nicht als Fachsimpel aufführen muß. Für sich selber nennt er Poesie und Literatur.

Im Kapitel «Arzt und Religion » wird auseinandergesetzt, daß die alten Philosophen im innersten Herzen schon Monotheisten gewesen seien und sich polytheistischer Formeln nur darum bedient hätten, um beim gemeinen Volk nicht unangenehm aufzufallen. Mit echter Herzensfrömmigkeit soll der Arzt jenem Glauben nachleben, von dem er überzeugt ist. Wie weit Scharandaeus seine Begriffe zu fassen pflegte, sehen wir in diesem Kapitel daran, daß unter Religion auch Kleider-, Haar- und Barttracht des Arztes abgehandelt werden, ebenso sein Verhalten gegenüber Wein und Weibern.

Scharandaeus lebte in der Zeit, da man begann, Astronomie und Astrologie auseinanderzuhalten. Er zitiert Copernicus und hält Erfolge der Astrologen in ihren Aussagen für das, was wir heute mit Zufallstreffer bezeichnen. Wo es um den vom Verfasser abgelehnten Einfluß des Mondes auf Krankheiten, namentlich auf die Anfallskrankheiten geht, lehnt er gleich auch den Zusammenhang zwischen Gezeiten und Mond ab. Von den Kalenderzeichen, unter denen zur Ader gelassen oder geschröpft werden solle, sei nichts zu halten. - Über das Klima des Ortes, an dem er praktiziert, soll der Arzt allerdings Bescheid wissen, und klimatischen Faktoren soll er bei der Beurteilung der Krankheitsphasen Rechnung tragen.

$\mathrm{Ob}$ die Behandlung im Altertum erfolgreicher gewesen sei als in der Gegenwart, gibt zunächst Gelegenheit zu einem Abriß der Medizingeschichte, für deren Anfänge er sich auf Plinius stützt; für spätere Zeiten wird die Quelle nicht genannt. Die Behandlung des Themas mündet schließlich in eine rhetorisch aufwendige erste Attacke gegen die Chymiker. Die Aufforderung des Petrus Severinus Danus, 
der als Gönner des Paracelsus hingestellt wird, die Natur in Feld und Wald genau zu erforschen, wird als sinnlos und hohl abgetan, weil sie darauf hinauslaufe, mit der Retorte zum Wesen der Dinge vorzustoßen.

Mit Ratschlägen zur Erhebung der Vorgeschichte einer Krankheit gelangt Scharandaeus endlich näher an das Verhältnis Arzt-Patient heran. Das Corpus Hippocraticum muß ausgiebig für Zitate herhalten. Gründlich werden die Fragen nach dem körperlichen Befinden dargestellt; Psychologisches beschränkt sich auf Bemerkungen über Simulanten. Breit eingegangen wird darauf, wie doppeldeutig das Volk die Bezeichnungen «Herz» und «Herzgegend» brauche.

Bemerkungen zum Pulsfühlen rufen zunächst einer Erörterung darüber, was «Leben» sei; für Scharandaeus ist es «Bewegung». Die Beziehung zwischen Bewegung und Wärme ist ihm bekannt, nicht aber der eigentliche Zusammenhang zwischen Leben, Bewegung und Wärmeproduktion. Daß im ganzen Kapitel Zitate kaum vorkommen, fällt auf, und wir wissen nicht, ob Scharandaeus den Namen und das Werk Harveys gekannt und die Entdeckung des Blutkreislaufs richtig gewürdigt hat. Wir sind nie ganz sicher, ob er «Vene» und «Arterie» auch funktionell genau auseinanderhält, auch wenn er meistens von «Puls des Herzens und der Arterien» spricht. Im praktischen Teil dieses Kapitels macht sich der Verfasser über spitzfindige Kollegen lustig, die sich anheischig machen, weiß der Himmel wie viele Pulsarten voneinander unterscheiden zu können. Scharandaeus hält nicht viel davon, bei der Prognosestellung besonders auf den Puls zu achten. «Bei Kopfkrankheiten wird der Schlag des Herzens und der Arterien in keiner Weise beeinflußt.» Weil der Autor wie auch seine Zeitgenossen noch keine Uhren mit Sekundenzeiger besaßen, übersahen sie die Bradykardie bei Hirndruck ${ }^{16}$. - Hier wird übrigens ganz kurz der einzige selbst beobachtete Fall gestreift, der im Büchlein überhaupt vorkommt.

Das Kapitel über die Harnschau wird eingeleitet mit einer Diskussion des Begriffs «Urin»: «Reinigungsprodukt des Blutes» möchte Scharandaeus als eigene Definition hinstellen. In der Auseinandersetzung mit dem von Hieronymus Mercurialis $^{17}$ erarbeiteten Harnbegriff wird darauf aufmerksam gemacht, daß Vögel trinken, aber keinen Urin produzieren, die Kaninchen aber nicht trinken und doch kräftig urinieren. (Was war doch noch alles zu entdecken in Physik, Chemie und Physiologie!) - Für den Akt der Harnschau selber verlangt Scharandaeus zunächst, daß die gesamte Harnmenge und nicht nur eine Probe aus einer Miktion vorliegen soll, und zwar nicht aufgerührt: «sic enim ea quae in imo, medio \& superficie sunt, optime perspicies.» Aber dann geht es ganz überraschend weiter mit «nec credas, ut ignari volunt, vas diaphanum semper requiri, quis enim vitrum apud omnes aegros transparens clarumque reperiet?». - Allzu 
kühne Schlußfolgerungen aus der Harnschau verwirft er - hier wohl beeinflußt von Langius -, zumal auch die Schwangerschaftsdiagnose ${ }^{11}$. Hingegen beklagt er sich über mannigfache Betrugsversuche seitens der Patienten. - Nach Darmausscheidungen soll sich der Arzt zwar erkundigen. Von Schleim- oder Blutbeimengung zu den Faeces ist aber nicht die Rede. Blut aus der «Goldader » galt ohnehin als günstiges Zeichen.

Im Abschnitt «Prognose» kommt Hippokrates wieder ausführlich zu Worte. Die Vorhersage sei vor allem auch dazu bestimmt, den Patienten dazu zu bewegen, daß er die Vorschriften des Arztes genau befolge. Hat sich ein Fall entgegen ungünstiger Prognose doch noch zum Guten gewendet, so soll der Arzt den Patienten ja nicht etwa als Lügner hinstellen wollen, so wenig wie Hippokrates schuld sein kann, wenn der Patient stirbt, obschon die klassischen Zeichen ungünstigen Ausgang nicht hatten erwarten lassen. Hier bildet die Philosophie den Schluß des Kapitels: Vorchristliche Autoren und der Araber Halys ${ }^{18}$ werden herbeibemüht, um zu sagen, ob die Dauer unseres Lebens von vorneherein und unabänderlich festgelegt sei. Wäre sie es, dann hinge jeder Versuch, es mit ärztlicher Kunst und Hilfe verlängern zu wollen, in der Luft.

Ein weiteres Kapitel vermittelt «Grundregeln der Behandlung, die für jedes Alter, jede Zeit, Gegend und Krankheit gelten ». Zwar kommen die einen Krankheiten in bestimmten Gegenden häufiger vor als in andern, wo andere Leiden vorherrschen; aber «der Saft im Leibe verursacht die Krankheit und nicht die Landesgegend». In diesem Abschnitt wird nur die Ableitung auf den Darm besprochen; sie soll nicht allzu brüsk oder mit allzu starken Mitteln bewerkstelligt werden und nie gleichzeitig nach oben und nach unten. Bereits fällt das Wort Medikamentenmißbrauch, und Scharandaeus verfolgt ihn weit über Jahrhunderte zurück.

Wie verschiedene Themen Scharandaeus im gleichen Kapitel behandeln konnte, zeigt das nächste: «Konsilien - Leichenschau - Zeugnisse». Mit viel Ironie beleuchtet er die Wirrnisse, die entstehen, wenn sich zwei Ärzte miteinander über ein Krankenbett beugen: Mangelhafte Orientierung, Besserwisserei auf beiden Seiten, aber auch ein Nichteingehen auf die Anamnese sind die Hauptanlässe dazu. Nicht verwunderlich ist es daher, daß schon Patienten gestorben sind, gerade weil über sie ein Konsilium abgehalten worden ist. Scharandaeus stellt Regeln auf, wie vorgegangen werden soll, damit der Patient den größtmöglichen Nutzen aus wirklicher Zusammenarbeit zweier vernünftiger Ärzte ziehen kann.

Große Zurückhaltung empfiehlt der Verfasser bei den Schlüssen, die der Arzt aus einer Leichenschau ableiten soll, einer Maßnahme, die ihm zuwider gewesen zu sein scheint. Wie man aber das Ansehen eines mutmaßlichen Selbstmörders 
und dessen Familie schonen kann (indem man nämlich gewaltsamen Tod durch fremde Hand in den Vordergrund rückt), gleichzeitig aber mutmaßliche Gewalttäter nicht belasten soll - dieser Widerspruch in seinen Empfehlungen scheint ihm nicht aufgegangen zu sein.

Auch mit gutachtlichen Äußerungen soll sich der Arzt nicht zu weit vorwagen. Im katholischen Solothurn scheinen Fragen über Impotenz und Sterilität an die Geistlichen, nicht an den Arzt gestellt worden zu sein wie im reformierten Basel Felix Platters ${ }^{19}$. Dagegen wurde der Arzt beigezogen bei Fragen nach der Tauglichkeit für den Eintritt in den geistlichen Stand oder für eine Beamtung, dies namentlich bei Sehschwachen und Anfallskranken. Hier rät Scharandaeus zur Vorsicht. Man lasse immer gewisse Zweifel an den eigenen Schlußfolgerungen durchschimmern!

Kauterisation gilt als beliebte und wirksame Maßnahme bei chronischen Krankheiten. Sie bei Hirnaffektionen am Arm vorzunehmen, hält Scharandaeus für aussichtslos.

Thermalwasser soll nur am Morgen auf den nüchternen Magen getrunken werden. Badekuren sind nur, wenn es genau angezeigt ist, und am geeigneten Ort zu verschreiben, und der Patient soll über alle Einzelheiten genau unterrichtet werden.

Bei der Besprechung des Aderlasses teilt Scharandaeus bereits die kritische Einstellung vieler Zeitgenossen, die sich auch in Ärztekreisen schon herausgebildet hatte, bevor 1673 Molière seinen «Malade imaginaire» auf die Bühne brachte. Er verwirft den Aderlaß bei Darmverschluß, Melancholie, Hypochondrie, Nierenentzündung, Schleimstühlen, Wassersucht, Icterus und Steinleiden und die Maßnahme überhaupt bei Kindern unter 7 Jahren, bei denen die Natur selber die Krankheiten heile. Gelten läßt er den Aderlaß im Beginn jener Krankheit, die wir heute als lobäre Pneumonie kennen, und freimütig gesteht er, nur der Volksmeinung zuliebe pflege er die Anordnung ein einziges Mal zu treffen, wenn er einen aufgeregten Geisteskranken oder einen Epileptiker zum erstenmal sehe. Ausgiebig wird erörtert, ob man mit Venaesectio eine Fehlgeburt hervorrufen könne und ob sich damit beim Ausbleiben der Periode aus anderer Ursache Entlastung verschaffen lasse.

Das Schröpfen scheint weniger mißbraucht worden zu sein als der Aderlaß. Schröpftage nach dem Kalender verwirft Scharandaeus ebenso wie die prophylaktische Wirkung der Maßnahme. Über die Blutegel wird heute noch Gültiges gesagt.

Wichtig war im 17.Jahrhundert, daß der Arzt die Phase richtig zu erkennen vermochte, in der sich eine Krankheit befand: Beginn, Anstieg, Höhepunkt, 
Abklingen, Heilung. Danach hatte er seine Maßnahmen zu richten. Scharandaeus läßt durchblicken, daß die Phasenlehre so gut wie die Konstitutionslehre bei den jungen Ärzten nicht mehr jene Beachtung fänden, die sie seiner Überzeugung nach verdienten. Betont wird die Notwendigkeit des Durchhaltens angeordneter Maßnahmen auch gegen Einwände von Angehörigen der Patienten.

Auch der «richtige Zeitpunkt» für besondere Maßnahmen wird wortreich besprochen. Wann ist Strenge und Beharren auf einmal getroffenen Anweisungen am Platz, und wann darf man die Zügel schleifen lassen? Hier wird auch diskutiert, was eigentlich eher ins Kapitel «Prognose» gehört hätte: Darf der Arzt zu Verstellung, ja Lüge Zuflucht nehmen, sofern er damit dem Kranken nützen kann? Merkwürdigerweise hier untergebracht ist auch die Warnung vor finanziellem Interesse an der Behandlung sowie vor Willfährigkeit und Unterwürfigkeit gegenüber vornehmen Patienten.

Es kommt vor, daß die Theorie und die Lehrbücher im Stiche lassen und daß das klassische Vorgehen versagt. Darf man dann auf Kühnheit abstellen und Gewagtes unternehmen? Autoren dafür und solche dagegen kommen zu Worte. Muß in einem Fall die Hoffnung auf Genesung ohnehin aufgegeben werden, so soll von Wagnissen abgesehen werden, um nicht ein Mittel zu diskreditieren, das ein andermal hilfreich sein könnte. Wenn überhaupt, dann soll metallisches Quecksilber bei Ileus rechtzeitig verabreicht werden.

Unter dem Titel «Bei Laien verbreitete Irrtümer» wird alles vorgelegt, was dem Arzt die Freude am Beruf vergällt, von den Simulanten bis zu den Besserwissern und den Leuten, die hinter des Doktors Rücken noch Kräuterweiber, Quacksalber und sogar den Henker aufsuchen und selbst dem Beichtvater noch Geheimmittel entlocken wollen, die sich anderswo als wirksam erwiesen hätten. Allemal heimst der Kurpfuscher die Lorbeeren ein, wenn der Kranke davonkommt, während ein übler Ausgang dem Arzte zur Last fällt.

Im Kapitel « Krankenkost » werden die schlimmen Folgen von Fraß und Völlerei anhand eines Zitates aus Seneca besprochen. Scharandaeus ist nicht unbedingter Anhänger zahlreicher Lehrbuchanweisungen; er verwirft aber Süßspeisen und kennt auch Nebenwirkungen von Medikamenten und Kostvorschriften. - Leuten, die an regelmäßigen Weingenuß gewöhnt sind, soll man ihn nicht schlagartig entziehen. Das Stichwort «delirium tremens» oder ein ähnlich klingendes fällt nicht.

Recht kurz äußert sich der Verfasser dazu, wie sich der Arzt gegenüber Patienten in den verschiedenen Lebensaltern verhalten soll.

Säuglinge und Kleinkinder überläßt er am besten den Ammen und den weisen Frauen. Kräftige Natur hilft ihnen meist aus der Krankheit wieder heraus. (Die 
Sterberegister jener Zeit lehren uns allerdings etwas anderes.) Medikamente vertragen sie ganz schlecht.

Mädchen gibt es nach Scharandaeus von zweierlei Art. Mit den einen darf man es nicht dadurch verderben, daß man ihnen Fragen nach den spezifischen Funktionen des weiblichen Körpers stellt; Großmütter, Erzieherinnen, Stubenmägde vermögen da vielleicht Auskunft zu geben, falls es unbedingt notwendig ist. Die andere Kategorie versucht, den Arzt zu täuschen und mit Klagen über Verstopfung zu starken Abführmitteln zu gelangen, um eine Leibesfrucht loszuwerden. Hier findet der Autor auch Anlaß, sich über die Verliebtheit zu äußern; er schließt sich Plato und dem Kirchenvater Laktanz an, wonach fleischliche Begierden ihren Sitz in der Leber haben.

Angenehmere Patienten sind die Männer: Man darf sie ungescheut über alles ausfragen und sogar körperlich untersuchen: «Eos enim et interrogare ultro quaecumque volueris atque etiam attrectare licet...» Allerdings neigen die Männer zu unmäßiger Lebensweise und sollten ihren Geschlechtstrieb besser zügeln. Gestützt auf eine Stelle aus Plato werden die Rauschkinder erwähnt. Hier zeigt sich Scharandaeus tief bekümmert über die weitverbreitete Einsichtslosigkeit gegenüber Empfehlungen zu vernünftigem Maßhalten; Apotheker und Ärzte sind dann die Nutznießer der üblen Folgen. - Männer pflegen Aufklärung über ihre Leiden zu verlangen. Wer auf alle Fragen gefaßt ist und entsprechende Antworten zu geben vermag, erwirbt sich den Ruf eines gescheiten und erfahrenen Arztes.

«Alle Krankheiten der Frauen haben irgendwie etwas mit der Gebärmutter zu tun », ist des Verfassers Extrakt aus den Werken seiner Gewährsleute Hippokrates, Varandaeus ${ }^{20}$, Rodericus a Castro, Daniel Sennert ${ }^{21}$. Recht unvermittelt kommt er auf die schwere Gebärmutterblutung - kaum auf die postpartale, eher auf die aus dem myomatösen Uterus - zu sprechen, gegen die man «unterhalb der falschen Rippen und nicht etwa oberhalb des Zwerchfells» eine Binde anlegen soll. Scharandaeus setzt ein Fragezeichen zu den Behauptungen, die menstruierende Frau mache Spiegel fleckig und Blumen verdorrten unter ihrer Hand. Nahrungsbeschränkung während der Schwangerschaft wird verworfen: «Der Foetus nimmt keine Nahrung zu sich.» In der Leitung der Geburt soll der Arzt vorsichtig sein und sich auf Anweisungen an die Hebamme beschränken. Ein Fehler kann seinem Ruf schwer schaden.

Führten wir in der Jugend einen vernünftigen und maßvollen Lebenswandel, dann wäre das Greisenalter weniger beschwerlich. Schon zu Scharandaeus' Zeiten muß es Nachkommen gegeben haben, die der Alten überdrüssig waren. Einzige Charakterveränderung, die erwähnt wird, ist der Geiz der Greise. Von ihnen 
unterscheiden sich die alten Weiber vor allem dadurch, daß ihre Geschlechtsfunktion erloschen ist und die Gebärmutter nicht mehr Anlaß zu Zwischenfällen gibt. Als triefnasige Wesen mit geröteten, dauernd tränenden Augen, bösartig im Umgang mit den Hausgenossen, dem Trunke ergeben, kommen sie beim Verfasser ganz schlecht weg.

An der Frage, ob es wirklich Mittel und Wege gebe, das Altern aufzuhalten und Jugendlichkeit zurückzugewinnen, entzündet sich neue Polemik gegen die Alchimisten. Einer der Beweise gegen die Alchimie, die solches versprach, lautet: Paracelsus ist ja auch nur 47 Jahre alt geworden.

75 «Sententiae communes» beschließen das Buch. Sie sind den Aphorismen des Hippokrates und anderer Schriftsteller nachgebildet. Zum Teil entsprechen sie Zusammenfassungen aus dem in den einzelnen Kapiteln Gesagten. Daraus sei die 51. herausgehoben, einmal des Irrtums wegen, der in der Ermahnung steckt: «Zweifle daran, daß man auf dem Weg über die Amme einen Säugling abführen kann. Es ist unglaubhaft, daß solche Medikamente in die Milch übergehen; sonst würden ja auch Gifte den gleichen Weg gehen, wenn sie von der Amme eingenommen werden », und zum andern des frappanten zweiten Teils der Sentenz wegen, der nun folgt: «und Greise würden auf diese Weise zur Darmentleerung veranlaßt, da sie auf Abführmittel leicht ansprechen », woraus wir erkennen, wie verbreitet damals der Gebrauch von Frauenmilch als Stärkungsmittel gewesen sein muß.

\section{Quellennachweis, Anmerkungen}

1 Auch Felix Platters Ausbildung zum Arzt war abgeschlossen, als er erst 20 Jahre zählte, wie uns sein Tagebuch (Basel, Schwabe, 1976, S.293 ff.) zeigt. Platter mußte nur befürchten, in diesem Alter noch nicht zur Doktorprüfung zugelassen zu werden. Ob Scharandaeus noch doktorieren konnte, als er dringend nach Hause gerufen wurde, scheint fraglich.

${ }^{2}$ Leu, Allg. Helvet. Lexikon XVIII, 314.

${ }^{3}$ Hist.-Biogr. Lexikon d. Schweiz VII, 70.

${ }^{4}$ Flüeler, B., Ärzte, Apotheker ... in Solothurn, 1481-1798, Diss. Basel 1951.

${ }^{5}$ Schubiger, F., Ärzte und Apotheker im alten Solothurn. In: Jb. f. Sol. Geschichte 8 (1935).

${ }^{6}$ Selten, nur in der Zentralbibliothek Solothurn vorhanden, die beiden andern Werke auch in den Zentralbibliotheken Luzern und Zürich.

${ }^{7}$ Leu, Lexikon XVI, 527 (Schwaller), und III, 40 (Bellelay).

${ }^{8}$ Haller, Albr., Bibliotheca Medica III, 7.

${ }^{9}$ Hollerius: Jacques Hollier († 1562). Nouv. Biogr. Gén. 25, 264.

10 Duretus: Jean Duret (1527-1586). Nouv. Biogr. Gén.15, 246. 
11 Johannes Langius (1485-1565): Allg. Deutsche Biogr.17, 637.

12 Valescus Tarentinus (15. Jh.): Hirsch, A. (Hrsg.), Lexikon berühmter Ärzte I, 302; N I, 60.

${ }^{13}$ Rodericus a Castro (1555-1624): Hirsch I, 855; N I, 164.

14 Platter, F., Praxeos ... tractatus III, erwähnt in Anm. 19.

15 Mani, N., in Festschrift Goerke, München 1978, S. 235.

16 Einer der ersten, der Pulszahlen pro Minute angab, war Carl Linné 1756. Clemedson, C. J., in Festschrift Goerke, München 1978, S.262.

${ }^{17}$ Hieronymus Mercurialis: Biogr. Univers. 28, 19.

${ }^{18}$ Halys, Ali-ben-Abbas († 995): Nouv. Biogr. Gén. 2, 96.

19 Platter, F., Observationes I (Hubers Klassiker der Medizin und der Naturwissenschaften, Bd.1), Bern 1963.

${ }^{20}$ Varandaeus: Hirsch $V$, 706; hier besonders «De morbis et affectibus mulierum libri III» $(1619,1620)$.

${ }^{21}$ Sennert, D. (1572-1637): Nouv. Biogr. Gén.43, 77.

\section{Summary}

Johann Jakob Scharandaeus (1630-1682), offspring of an aristocratic family of Solothurn, has been physician and medical officer of that town since 1650. After 20 years of practicing he published a booklet of 280 pages "Modus et ratio visendi aegros" whose purpose was to advise young doctors beginning with their activity. Although he was a strict follower of Hippocrate and Galenus, he appears unsatisfied by many conclusions derived from uroscopy and the qualities of the pulse. Patients can be very disappointing to their doctors, as can be many colleagues on the occasion of a consultation. Reserved towards venaesectio, he tends his patients according to Galenus and Celsus and to his former teachers at the university in Montpellier. He is strictly opposed to Paracelsus and the alchemists, as well as to more modern philosophers like Descartes. The volume shows the state of knowledge of an average doctor living between 1650 and 1680 .

Dr. med. Joseph Schürmann-Roth

Bergstraße 24

CH-6004 Luzern 